Article

\title{
Weak Solution for 3D-Stochastic Third Grade Fluid Equations
}

\author{
Adilson Almeida (1) and Fernanda Cipriano * (i)
}

Faculdade de Ciências e Tecnologia da Universidade Nova de Lisboa and Centro de Matemática e Aplicações, Largo da Torre, 2825149 Caparica, Portugal; ama.almeida@campus.fct.unl.pt

* Correspondence: cipriano@fct.unl.pt

Received: 20 October 2020; Accepted: 12 November 2020; Published: 16 November 2020

check for updates

\begin{abstract}
This article studies the stochastic evolution of incompressible non-Newtonian fluids of differential type. More precisely, we consider the equations governing the dynamic of a third grade fluid filling a three-dimensional bounded domain $\mathcal{O}$, perturbed by a multiplicative white noise. Taking the initial condition in the Sobolev space $H^{2}(\mathcal{O})$, and supplementing the equations with a Navier slip boundary condition, we establish the existence of a global weak stochastic solution with sample paths in $L^{\infty}\left(0, T ; H^{2}(\mathcal{O})\right)$.
\end{abstract}

Keywords: non-Newtonian fluid; stochastic partial differential equation; third grade fluid; turbulent flow

\section{Introduction}

The incompressible Newtonian fluids described by the Navier-Stokes equation are characterized by the Newton law of the viscosity, which corresponds to a linear relation between the shear stress and the rate-of-strain tensors. However, some biological fluids (as the blood) and many fluids used in the industry and in the food processing do not satisfy such linear relation and are named non-Newtonian fluids (see for instance [1-5]).

The fluids of grade $n$ constitute a special class of non-Newtonian fluids. For these fluids the stress tensor corresponds to a polynomial of degree $n$ of the first $n$ Rivlin-Ericksen kinematic tensors in [6] $\mathbf{A}_{1}, \ldots, \mathbf{A}_{n}$,

$$
\begin{gathered}
\mathbf{A}_{1}(y)=\nabla y+(\nabla y)^{\top}, \\
\mathbf{A}_{k}(y)=\frac{d}{d t} \mathbf{A}_{k-1}(y)+\mathbf{A}_{k-1}(y)(\nabla y)+(\nabla y)^{\top} \mathbf{A}_{k-1}(y), \quad k=1, \ldots, n,
\end{gathered}
$$

where $y$ denotes the velocity vector field.

The Cauchy stress tensor is given by

$$
\mathbb{T}=\mathbb{T}_{1}+\mathbb{T}_{2}+\mathbb{T}_{3}
$$

where

$$
\begin{aligned}
& \mathbb{T}_{1}(y)=-p \mathbb{I}+v \mathbf{A}_{1}(y), \\
& \mathbb{T}_{2}(y)=\alpha_{1} \mathbf{A}_{2}(y)+\alpha_{2} \mathbf{A}_{1}^{2}(y), \\
& \mathbb{T}_{3}(y)=\beta_{1} \mathbf{A}_{3}(y)+\beta_{2}\left(\mathbf{A}_{1}(y) \mathbf{A}_{2}(y)+\mathbf{A}_{2}(y) \mathbf{A}_{1}(y)\right)+\beta_{3}\left(\operatorname{tr} \mathbf{A}_{1}^{2}(y)\right) \mathbf{A}_{1}(y),
\end{aligned}
$$

here $\frac{d}{d t}=\frac{\partial}{\partial t}+y \cdot \nabla$ stands for the material derivative, $p$ is the pressure, $\mathbb{I}$ is the unit tensor, and $\alpha_{1}, \alpha_{2}$, $\beta_{1}, \beta_{2}$ and $\beta_{3}$ are material moduli. 
The momentum equation with an external deterministic body force $F$ depending on the solution reads

$$
\frac{d y}{d t}=\operatorname{div} \mathbb{T}(y)+F(t, y) .
$$

Physical considerations in $[1,2]$ impose the following restrictions on the parameters

$$
v \geq 0, \quad \alpha_{1} \geq 0, \quad\left|\alpha_{1}+\alpha_{2}\right| \leq \sqrt{24 v \beta_{3}}, \quad \beta_{1}=\beta_{2}=0, \quad \beta_{3} \geq 0 .
$$

We set $\beta=\beta_{3}$.

Fluids of third grade are complex fluids that belong to the class of differential type viscoelastic fluids and arise in polymer processing, coating, colloidal suspensions and emulsions, ink-jet prints, geological flows, etc.; its mathematical analysis is crucial to predict and control its behavior, in order to design optimal flows that can be successfully used and applied in the industry.

In the deterministic theory of the fluids dynamic, the momentum Equation (4) being a partial differential equation can be supplemented with a boundary condition, in order to have a solution for each initial condition in a suitable space of vector fields. The most used boundary condition is the Dirichlet boundary condition, which corresponds to assume that the particles adjacent to the boundary surface have the same velocity as the boundary. However practical studies show that some viscoelastic fluids slip against the boundary surface. Let us refer, for instance, the articles [7] on capillary flow of highly entangled polyethylene (PE) melts, and [8] on microgel pastes and concentrated emulsions exhibiting a generic slip behavior at low stresses when sheared near smooth surfaces. In this work, we consider an homogeneous slip boundary condition.

Essentially, the deterministic approach consists of the study of well-posedness and properties of an individual solution, which describes quite well the laminar flows of fluids. However, most flows of fluids existing in nature and used in industry have a turbulent behavior; and it is widely recognized that the accurate description of turbulent flows requires a statistical approach, which relays on the study of properties of ensembles of flows of fluids (see [9-12]).

In this framework, the flows of fluids are described by stochastic processes with values in appropriate Sobolev spaces. Individual flows correspond to the realizations of the corresponding stochastic process. From this perspective, we add to the momentum equation a multiplicative white noise of Itô type, and describe the evolution of the velocity field by the following stochastic partial differential equation

$$
\frac{d Y}{d t}=\operatorname{div} \mathbb{T}(Y)+F(t, Y)+\sigma(t, Y) \frac{d \mathcal{W}}{d t} .
$$

The stochastic perturbation of the momentum equation induces the appropriate randomness of the evolutionary velocity field in order to model the intrinsic random effects of the turbulent dynamic, as well as the external random disturbances of the physical system.

Other relevant stochastic perturbation can be performed by adding to the deterministic equation an additive or a multiplicative white noise of Stratonovich type $\sigma(t, Y) \circ \frac{d \mathcal{W}}{d t}$. The nature of the two stochastic perturbations is different and the practical choice of the more appropriate one to describe the features of the physical system is not an easy issue. From our perspective the drawback of the Stratonovich perturbation is not to be a purely stochastic perturbation, it can be written as a sum of an Itô perturbation plus a deterministic perturbation, which clearly modifies the deterministic part of the system. In the present work, we have chosen to deal with an Itô type perturbation maintaining the deterministic part of the model. One of our main motivations relays on the application of the Large deviation principle, in order to identify and to compute the probability of some specific rare events, which can highlight the onset of turbulent behavior of the fluid. However, there are mathematical and physical reasons that justify future research on the three dimensional stochastic third grade fluid equations with a Stratonovich type perturbation, namely the Wong-Zakai principle and the existence of stochastic variational principles $[13,14]$. 
Introducing the expression of the Cauchy tensor $\mathbb{T}$ in Equation (6), we arrive at the following stochastic partial differential equation

$$
\begin{aligned}
d v(Y(t))= & \left(-\nabla p+v \Delta Y-(Y \cdot \nabla) v-\sum_{j} v^{j} \nabla Y^{j}+\left(\alpha_{1}+\alpha_{2}\right) \operatorname{div}\left(A^{2}\right)\right. \\
& \left.+\beta \operatorname{div}\left(|A|^{2} A\right)+F(t, Y)\right) d t+\sigma(t, Y) d \mathcal{W}(t) \quad \operatorname{in} \mathcal{O} \times(0, T),
\end{aligned}
$$

where

$$
\begin{gathered}
A=A(Y)=\mathbf{A}_{1}(Y)=2 D(Y) \quad \text { with } \quad D(Y)=\frac{1}{2}\left[\nabla Y+\nabla Y^{\top}\right] \\
v=v(Y)=Y-\alpha_{1} \Delta Y
\end{gathered}
$$

and $\sigma(t, Y)$ is the diffusion coefficient.

In the case $\beta=0$, the third grade fluid equations reduce to the second grade fluid equations, which are mathematically more tractable (see [15-17]). Nevertheless, in order to capture important rheological properties of certain fluids, as for instance the shear thinning and shear thickening effects, we need to deal with a third grade fluid model.

As far as we know, the stochastic third grade fluid equations have been studied for the first time in the article [18], where the authors proved the existence and the uniqueness of the strong solution (in the stochastic sense) in dimension two. The strategy applied in [18] is based on a combination of the deterministic methods developed in the articles $[19,20]$ with a stochastic uniqueness type argument introduced in [21] to study the stochastic Navier-Stokes equation. We should mention that the strategy in [18] does not work in dimension three.

\section{Results}

In this article, we address the physically relevant three-dimensional problem. Having in mind the statistical study of turbulent flows, the solution of the stochastic momentum equation is defined as a stochastic process with values in the Sobolev space of the initial conditions. In Section 2, we formulate the problem by adding a Navier slip boundary condition to the equation, and introducing the appropriate functional spaces. The Section 3 constitutes the main part of the article. We present the notion of a weak solution (in the probabilistic sense), with the Wiener process being part of the solution, and establish its existence, for initial conditions in the Sobolev space $H^{2}(\mathcal{O})$. We prove that the realizations of the stochastic process of the velocity field live in $L^{\infty}\left(0, T ; H^{2}(\mathcal{O})\right)$. The methods for showing existence are based on the Galerkin's finite-dimensional approximations and on the application of Prokhorov's and Skorohod's Theorems.

\section{Functional Setting and Useful Results}

We consider the stochastic third grade fluid Equation (7), with $\beta>0$, in a bounded, non-axisymmetric and simply connected domain $\mathcal{O}$ of $\mathbb{R}^{3}$ with a sufficiently regular boundary $\Gamma$, and supplemented with a Navier slip boundary condition, which reads

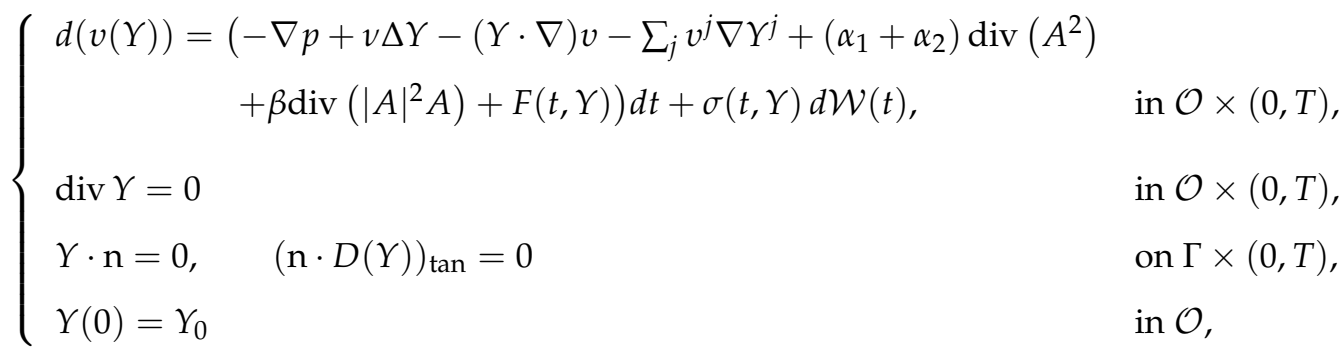


where $Y=\left(Y_{1}, Y_{2}, Y_{3}\right)$ is the fluid velocity field, $\mathrm{n}=\left(n_{1}, n_{2}, n_{3}\right)$ is the unit normal to the boundary $\Gamma$, $(\mathrm{n} \cdot D(Y))_{\tan }$ denotes the tangent part of the vector $\mathrm{n} \cdot D(Y)$, and the term $\sigma(t, Y) d \mathcal{W}(t)$ corresponds to the stochastic perturbation, where $\mathcal{W}(t)$ is a standard $\mathbb{R}^{m}$-valued Wiener process defined on a stochastic basis $\left(\Omega, \mathcal{F}, P,\left\{\mathcal{F}_{t}\right\}_{t \in[0, T]}\right)$. We assume that $\mathcal{F}_{0}$ is a complete $\sigma$-algebra with respect to $P$.

Taking an open and bounded subset $\mathcal{O}$ of $\mathbb{R}^{3}$, we consider the Lebesgue spaces $L^{p}(\mathcal{O})$ and the Sobolev spaces $W^{j, p}(\mathcal{O})$ endowed with the usual norms denoted by $\|\cdot\|_{p}$ and $\|\cdot\|_{W^{j, p}}$, respectively, $j \in \mathbb{N}, p>0$. In the particular case $p=2$, the spaces $W^{j, p}(\mathcal{O})$ and the norms $\|\cdot\|_{W^{j, p}}$ will be denoted by $H^{j}(\mathcal{O})$ and $\|\cdot\|_{H^{j}}$, respectively.

We introduce the following Hilbert spaces:

$$
\begin{aligned}
& H=\left\{y \in L^{2}(\mathcal{O}): \operatorname{div} y=0 \text { in } \mathcal{O} \text { and } y \cdot \mathrm{n}=0 \text { on } \Gamma\right\} \\
& V=\left\{y \in H^{1}(\mathcal{O}): \operatorname{div} y=0 \text { in } \mathcal{O} \text { and } y \cdot \mathrm{n}=0 \text { on } \Gamma\right\} \\
& W=\left\{y \in V \cap H^{2}(\mathcal{O}):(\mathrm{n} \cdot D(y))_{\tan }=0 \text { on } \Gamma\right\} .
\end{aligned}
$$

The space $H$ is endowed with the usual $L^{2}$-inner product $(\cdot, \cdot)$ and the associated norm $\|\cdot\|_{2}$.

Let us recall the Helmholtz-Leray projector $\mathbb{P}: L^{2}(\mathcal{O}) \longrightarrow H$, which is the linear bounded operator characterized by the following $L^{2}$-orthogonal decomposition of vector fields

$$
z=\mathbb{P} z+\nabla \phi, \quad \phi \in H^{1}(\mathcal{O}) .
$$

Due to the structure of Equation (7), it is convenient to introduce on $V \subset H^{1}(\mathcal{O})$ the following inner product

$$
(u, z)_{V}:=(v(u), z)=(u, z)+2 \alpha_{1}(D(u), D(z)),
$$

and define the associated norm $\|\cdot\|_{V}$, which is equivalent to the norm $\|\cdot\|_{H^{1}}$. Analogously, on $W \subset$ $H^{1}(\mathcal{O})$, we introduce the inner product

$$
(u, z)_{W}:=(u, z)_{V}+(\mathbb{P} v(u), \mathbb{P} v(z))
$$

and denote by $\|\cdot\|_{W}$ the corresponding norm, which is equivalent to the norm $\|\cdot\|_{H^{2}}$.

Throughout the article, we denote by $\mathrm{C}$ a generic positive constant that can assume different values from line to line.

We consider the following auxiliary modified Stokes problem with Navier boundary condition

$$
\begin{cases}\tilde{f}-\alpha_{1} \Delta \tilde{f}=f-\nabla p, \quad \operatorname{div} \tilde{f}=0 & \text { in } \mathcal{O}, \\ \tilde{f} \cdot \mathrm{n}=0, \quad(\mathrm{n} \cdot D(\tilde{f}))_{\tan }=0 \quad & \text { on } \Gamma .\end{cases}
$$

We recall from [16] that assuming $f \in H^{m}(\mathcal{O}), m=0,1$, the problem (12) has a solution $(\tilde{f}, p) \in$ $H^{m+2}(\mathcal{O}) \times H^{m+1}(\mathcal{O})$ verifying

$$
\|\tilde{f}\|_{H^{2}} \leq C\|f\|_{2} .
$$

According to the definition of the inner product (10), we have

$$
(\tilde{f}, h)_{V}=(f, h), \quad \forall h \in V .
$$

Let $X$ be a real Banach space endowed with the norm $\|\cdot\|_{X}$. We denote by $L^{p}(0, T ; X)$ the space of $X$-valued measurable $p$-integrable functions $y$ defined on $[0, T]$ for $p \geq 1$. 
Given $\eta \in(0,1), q \in(1, \infty)$, we consider the space $W^{\eta, q}(0, T ; X)$ endowed with the norm

$$
\|y\|_{W^{\eta, q}(0, T ; X)}^{q}=\|y\|_{L^{q}(0, T ; X)}^{q}+\int_{0}^{T} \int_{0}^{T} \frac{\|y(t)-y(s)\|_{X}^{q}}{|t-s|^{1+\eta q}} d t d s .
$$

If $\eta q>1$, we have the Sobolev's continuous embedding

$$
W^{\eta, q}(0, T ; X) \subset C([0, T], X) .
$$

The Ascoli-Arzelá's Theorem gives the next compactness result.

Lemma 1. Let $X_{0}$ and $X$ be Banach spaces with the compact embedding $X_{0} \hookrightarrow X$. Then for all $\eta \in(0,1)$, $p \in(1, \infty)$, such that $\eta p>1$, the following compact embedding holds

$$
W^{\eta, p}\left(0, T ; X_{0}\right) \hookrightarrow C([0, T], X) .
$$

For the next lemma, we refer [22].

Lemma 2. Let us consider Banach spaces $X_{0} \subset X \subset X_{1}$. Assume that the first inclusion is compact. Then for $\eta \in(0,1)$ and $p \in(1, \infty)$, the space

$$
L^{p}\left(0, T ; X_{0}\right) \cap W^{\eta, p}\left(0, T ; X_{1}\right)
$$

with the norm

$$
\|y\|_{\eta, p, X_{0}, X_{1}}:=\|y\|_{L^{p}\left(0, T ; X_{0}\right)}+\|y\|_{W^{\eta, p}\left(0, T ; X_{1}\right)}
$$

is campactly embedded in the space $L^{p}(0, T ; X)$.

For $p, r \geq 1$ let $L^{p}\left(\Omega, L^{r}(0, T ; X)\right)$ be the space of stochastic processes $Y=Y(\omega, t)$ with values in $X$ defined on $\Omega \times[0, T]$, adapted to the filtration $\left\{\mathcal{F}_{t}\right\}_{t \in[0, T]}$, and endowed with the norms

$$
\|Y\|_{L^{p}\left(\Omega, L^{r}(0, T ; X)\right)}=\left(\mathbb{E}\left(\int_{0}^{T}\|Y\|_{X}^{r} d t\right)^{\frac{p}{r}}\right)^{\frac{1}{p}}
$$

and

$$
\|Y\|_{L^{p}\left(\Omega, L^{\infty}(0, T ; X)\right)}=\left(\mathbb{E} \sup _{t \in[0, T]}\|Y\|_{X}^{p}\right)^{\frac{1}{p}} \quad \text { if } r=\infty,
$$

where $\mathbb{E}$ is the mathematical expectation with respect to the probability measure $P$.

\section{Regularity Assumptions}

We consider a deterministic initial condition $Y_{0} \in W$. In addition, we assume that the deterministic force $F:[0, T] \times V \rightarrow L^{2}(\mathcal{O})$, as well as the diffusion coefficient

$$
\sigma:[0, T] \times V \rightarrow\left(L^{2}(\mathcal{O})\right)^{m}, \quad \sigma(t, y)=\left(\sigma^{1}(t, y), \ldots, \sigma^{m}(t, y)\right),
$$

satisfy a growth condition and are Lipschitz, namely there exist positive constants $L, K$ and $0 \leq \gamma<2$ such that

$$
\begin{gathered}
\|F(t, y)\|_{2}^{2} \leq L\left(1+\|y\|_{W^{1,4}}^{\gamma}\right), \quad \forall y \in W^{1,4}(\mathcal{O}) \cap V, \\
\|F(t, y)-F(t, z)\|_{2}^{2} \leq K\|y-z\|_{V}^{2}, \quad \forall y, z \in V, t \in[0, T],
\end{gathered}
$$


and

$$
\begin{gathered}
\|\sigma(t, y)\|_{2}^{2} \leq L\left(1+\|y\|_{W^{1,4}}^{\gamma}\right), \quad \forall y \in W^{1,4}(\mathcal{O}) \cap V, \\
\|\sigma(t, y)-\sigma(t, z)\|_{2}^{2} \leq K\|y-z\|_{V}^{2}, \quad \forall y, z \in V, t \in[0, T],
\end{gathered}
$$

where we consider that

$$
\|\sigma(t, y)\|_{2}^{2}:=\sum_{i=1}^{m}\left\|\sigma^{i}(t, y)\right\|_{2}^{2}
$$

Analogously if $\sigma^{i}(t, y) \in V$ or $\sigma^{i}(t, y) \in W, i=1, \ldots, m$, we write

$$
\|\sigma(t, y)\|_{V}^{2}:=\sum_{i=1}^{m}\left\|\sigma^{i}(t, y)\right\|_{V}^{2}, \quad\|\sigma(t, y)\|_{W}^{2}:=\sum_{i=1}^{m}\left\|\sigma^{i}(t, y)\right\|_{W}^{2} .
$$

We also introduce the notation

$$
(\sigma(t, y), z):=\left(\left(\sigma^{1}(t, y), z\right), \ldots,\left(\sigma^{m}(t, y), z\right)\right) \quad \forall y \in V, z \in L^{2}(\mathcal{O}) .
$$

Accordingly

$$
|(\sigma(t, y), z)|=\left(\sum_{k=1}^{m}\left(\sigma^{k}(t, y), z\right)^{2}\right)^{1 / 2}, \quad \forall y \in V, z \in L^{2}(\mathcal{O}) .
$$

Remark 1. Let us emphasize that the assumptions (18)-(19) allow diffusion coefficients depending on a power less that $\frac{1}{2}$ of the first order derivatives of the velocity field $y$, with any intensity.

However, there are specific diffusion coefficients that do not satisfy the above assumptions, for which we can still apply the same strategy to show the existence of the solution. This is the case for

$$
\sigma(t, y)=\sigma_{0} v(y)
$$

where $\sigma_{0}$ is a constant small enough compared to the parameter $\beta$. The key point is that in the deduction of the energy estimates, we can take advantage of the $L^{2}$-symmetry of $\sigma(t, y)$ through the integration by parts, as for instance

$$
(\sigma(t, y), y)=\sigma_{0}(v(y), y)=\sigma_{0}\|y\|_{V}^{2} \leq C \sigma_{0}\|y\|_{W^{1,4}}^{2} .
$$

We should mention that the addition of this type of stochastic noises to the deterministic equations, may have relevant physical consequences, namely it helps to stabilize the physical system, as $t \rightarrow \infty$.

\section{Existence of Solution}

This section is devoted to show the existence of the solution to the stochastic system (8). Let us first introduce the notion of the solution.

Definition 1. Assume the hypothesis (16)-(19) and consider $Y_{0} \in W$. A system

$$
\left(\bar{\Omega}, \overline{\mathcal{F}}, \bar{P},\left\{\overline{\mathcal{F}}_{t}\right\}_{t \in[0, T]}, \overline{\mathcal{W}}, \bar{Y}\right)
$$

is a stochastic weak solution of Equation (8) if

1. $(\bar{\Omega}, \overline{\mathcal{F}}, \bar{P})$ is a complete probability space;

2. $\overline{\mathcal{W}}$ is a Brownian motion defined on the probability space $(\bar{\Omega}, \overline{\mathcal{F}}, \bar{P})$ for the filtration $\left\{\overline{\mathcal{F}}_{t}\right\}_{t \in[0, T]}$;

3. for a.e. $t, \bar{Y}(t)$ is $\overline{\mathcal{F}}_{t}-$ measurable and belongs to $L^{p}\left(\bar{\Omega}, L^{\infty}(0, T ; W)\right)$ for every $2 \leq p<\infty$; 
4. a.e. $-\bar{P}$ the following integral equation holds

$$
\begin{aligned}
(v(\bar{Y}(t)), \phi) & =\int_{0}^{t}\left[v(\Delta \bar{Y}, \phi)-((\bar{Y} \cdot \nabla) \phi, v(\bar{Y}))-\sum_{j}\left(v^{j}(\bar{Y}) \nabla \bar{Y}^{j}, \phi\right)\right] d s \\
& -\int_{0}^{t}\left(\left(\alpha_{1}+\alpha_{2}\right) \operatorname{div}\left(\bar{A}^{2}\right)+\beta \operatorname{div}\left(|\bar{A}|^{2} \bar{A}\right), \phi\right) d s \\
& +\left(v\left(Y_{0}\right), \phi\right)+\int_{0}^{t}(F(s, \bar{Y}(s)), \phi) d s+\int_{0}^{t}(\sigma(s, \bar{Y}(s)), \phi) d \overline{\mathcal{W}}(s),
\end{aligned}
$$

$\forall \phi \in V, \forall t \in[0, T]$, where the stochastic integral is defined by

$$
\int_{0}^{t}(\sigma(s, \bar{Y}(s)), \phi) d \overline{\mathcal{W}}(s)=\sum_{k=1}^{m} \int_{0}^{t}\left(\sigma^{k}(s, \bar{Y}(s)), \phi\right) d \overline{\mathcal{W}}^{k}(s),
$$

and $\bar{A}:=A(\bar{Y})$.

Now we state the main result of the article.

Theorem 1. Assume the hypothesis (16)-(19) and consider $Y_{0} \in W$. Then there exists a solution $\left(\bar{\Omega}, \overline{\mathcal{F}}, \bar{P},\left\{\overline{\mathcal{F}}_{t}\right\}_{t \in[0, T]}, \overline{\mathcal{W}}, \bar{Y}\right)$ to Equation (8) according to the Definition 1.

The remaining of this section is devoted to the proof of Theorem 1. As in the articles [18,20,23], we apply the Galerkin's approximation method for an appropriate basis. We recall that the injection operator $I: W \hookrightarrow V$ being a compact operator guarantees the existence of a basis $\left\{e_{i}\right\} \subset W$ of eigenfunctions to the problem

$$
\left(v, e_{i}\right)_{W}=\lambda_{i}\left(v, e_{i}\right)_{V}, \quad \forall v \in W, \quad i \in \mathbb{N},
$$

which is an orthonormal basis in $V$ and an orthogonal basis in $W$. In addition the sequence $\left\{\lambda_{i}\right\}$ of the corresponding eigenvalues fulfils the properties: $\lambda_{i}>0, \forall i \in \mathbb{N}$, and $\lambda_{i} \rightarrow \infty$ as $i \rightarrow \infty$. Since the ellipticity of Equation (21) increases the regularity of their solutions (see [24]), we may consider $\left\{e_{i}\right\} \subset H^{4}(\mathcal{O})$.

We consider the finite dimensional space $W_{n}=\operatorname{span}\left\{e_{1}, \ldots, e_{n}\right\}$, and introduce the Faedo-Galerkin approximation of the system (8). Namely, we look for a solution to the following stochastic differential equation

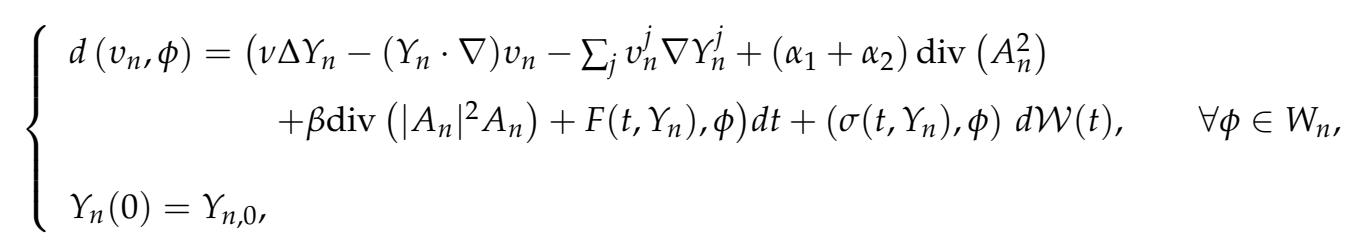

where

$$
Y_{n}(t)=\sum_{j=1}^{n} c_{j}^{n}(t) e_{j}
$$

Here $Y_{n, 0}$ denotes the projection of the initial condition $Y_{0}$ onto the space $W_{n}, v_{n}=Y_{n}-\alpha_{1} \Delta Y_{n}$ and $A_{n}=\nabla Y_{n}+\left(\nabla Y_{n}\right)^{\top}$.

Due to the relation (21), the sequence $\left\{\widetilde{e}_{j}=\frac{1}{\sqrt{\lambda_{j}}} e_{j}\right\}$ is an orthonormal basis for $W$ and

$$
Y_{n, 0}=\sum_{j=1}^{n}\left(Y_{0}, e_{j}\right)_{V} e_{j}=\sum_{j=1}^{n}\left(Y_{0}, \widetilde{e}_{j}\right)_{W} \widetilde{e}_{j}
$$


The Parseval's identity yields

$$
\left\|Y_{n}(0)\right\|_{V} \leq\left\|Y_{0}\right\|_{V} \quad \text { and } \quad\left\|Y_{n}(0)\right\|_{W} \leq\left\|Y_{0}\right\|_{W} .
$$

The Equation (22) can be written as a system of stochastic ordinary differential equations in $\mathbb{R}^{n}$ with locally Lipschitz nonlinearities. From classical results there exists a local-in-time solution $Y_{n}$ that is an adapted stochastic process with values in $C\left(\left[0, T_{n}\right], W_{n}\right)$.

The existence of a global-in-time solution follows from the uniform estimates on $n=1,2, \ldots$, that will be established in the next section (a similar reasoning can be found in $[16,17,25])$.

If we identify the Hilbert space $V$ with its dual $V^{*}$, we have the Gelfand triplet

$$
W \subset V=V^{*} \subset W^{*} .
$$

The duality relations between $u \in W^{*}$ and $y \in W$ is denoted by the inner product $(u, y)_{V}$ in $V$, in the case of $u \in V$ the duality coincide with the inner product in $V$.

\subsection{Uniform Estimates}

Here, we state two lemmas, which have been proved in the articles $[18,23]$ within the two-dimensional context. By performing straightforward adaptation, all steps of the proofs remain valid if we consider $\mathcal{O} \subset \mathbb{R}^{3}$.

Lemma 3 ([18]). Assume (16)-(19), and $Y_{0} \in W$. The solution $Y_{n}$ to Equation (22) verifies

$$
\begin{gathered}
\mathbb{E} \sup _{t \in[0, T]}\left\|Y_{n}\right\|_{V}^{p} \leq C, \\
\mathbb{E} e^{\frac{\beta}{16(K *)^{4}} \int_{0}^{T}\left\|Y_{n}\right\|_{W^{1,4}}^{4} \leq C,} \\
\mathbb{E} \sup _{t \in[0, T]}\left\|Y_{n}\right\|_{W}^{p} \leq C,
\end{gathered}
$$

for every $2 \leq p<\infty$, where $C$ is a constant independent of $n$, and $K_{*}$ is defined by the following inequality

$$
\|y\|_{W^{1,4}} \leq K_{*}\|A(y)\|_{4}, \quad \forall y \in V .
$$

Let us notice that the inequality (26) is a consequence of the following version of the Korn inequality, which holds for non-axisymmetric bounded domains (see Theorem 3 in [26])

$$
\|\nabla y\|_{2} \leq K\|A(y)\|_{2}, \quad \forall y \in V,
$$

where $K$ is a positive constant, conjugated with the Sobolev's continuous embeddings $H^{1}(\mathcal{O}) \hookrightarrow$ $L^{4}(\mathcal{O}) \hookrightarrow L^{2}(\mathcal{O})$ and the Poincaré inequality.

The main estimate (24) follows from the key remark that the nonlinear term $\beta \operatorname{div}\left(|A(y)|^{2} A(y)\right)$ for $\beta>0$ is more regularizing than the linear term $v \Delta y$, due to its symmetry and good sign. Namely, it helps to absorb the other inconvenient nonlinear terms, in order to deduce the inequality

$$
\begin{aligned}
\left\|Y_{n}(t)\right\|_{V}^{2} & +\frac{\beta}{4} \int_{0}^{t}\left\|A\left(Y_{n}\right)\right\|_{4}^{4} d s+4 v \int_{0}^{t}\left\|D Y_{n}\right\|_{2}^{2} d s \leq\left\|Y_{0}\right\|_{V}^{2} \\
& +C\left(1+\int_{0}^{t}\left\|Y_{n}\right\|_{V}^{2} d s\right)+2 \int_{0}^{t}\left(\sigma\left(s, Y_{n}\right), Y_{n}\right) d \mathcal{W}_{s}, \quad \forall t \in[0, T]
\end{aligned}
$$

where $C$ is a positive constant depending on the parameters of the equation. Next, by using standard Sobolev's inequalities, the inequality (26) and the exponential supermartingale process, we infer (24). 
Lemma 4 ([23]). Assume (16)-(19), and $Y_{0} \in W$. Let $Y_{n}$ be the unique solution to Equation (22). Then for $2 \leq p<\infty$ and $0<\eta<\frac{1}{2}$, we have

$$
\mathbb{E}\left\|Y_{n}\right\|_{W^{\eta, p}(0, T ; V)}^{p} \leq C
$$

where $C$ is a positive constant independent of $n$.

These uniform estimates are crucial to apply a suitable compactness criterion, in order to pass to the limit the sequence $\left\{Y_{n}\right\}$ of Galerkin approximations.

\subsubsection{Tightness} the set

Let us introduce the stochastic process $\Psi_{n}(t):=\left(Y_{n}(t), \mathcal{W}(t)\right)$. For $1 \leq q \leq 6$ fixed, we define

$$
\mathcal{E}:=L^{2}\left(0, T, W^{1, q}(\mathcal{O})\right) \cap C([0, T], H) .
$$

The trajectories of the stochastic processes $\Psi_{n}(t)$ belong to the space

$$
\mathcal{E} \times C\left([0, T], \mathbb{R}^{m}\right) .
$$

The laws $\mu_{n}, n \in \mathbb{N}$, of the random variables

$$
\Psi_{n}: \Omega \rightarrow \mathcal{E} \times C\left([0, T], \mathbb{R}^{m}\right)
$$

are defined by

$$
\mu_{n}(B)=P\left(\Psi_{n} \in B\right) \quad \text { for any Borel set } B \in \mathcal{B}\left(\mathcal{E} \times C\left([0, T], \mathbb{R}^{m}\right)\right)
$$

For any $R>0$, let us consider the set

$$
K_{R}:=\left\{y \in L^{2}(0, T ; W) \cap W^{\gamma, 2}(0, T ; V):\|y\|_{\gamma, 2, W, V} \leq R\right\},
$$

with the norm $\|\cdot\|_{\gamma, 2, W, V}$ defined in (15). Since the embedding $V \hookrightarrow H$ is compact, Lemma 1 gives the compact embedding

$$
W^{\gamma, p}(0, T ; V) \hookrightarrow C([0, T], H) ;
$$

and Lemma 2 yields the compact embedding

$$
L^{2}(0, T ; W) \cap W^{\gamma, 2}(0, T ; V) \hookrightarrow L^{2}\left(0, T ; W^{1, q}(\mathcal{O})\right) \text { for any } \quad 2<q \leq 6 .
$$

Therefore, the set $K_{R}$ is a relatively compact subset of $\mathcal{E}$.

Due to estimate (25) of Lemma 3 and Lemma 4

$$
P\left(Y_{n} \notin K_{R}\right)=P\left(\left\|Y_{n}\right\|_{\gamma, 2, W, V}>R\right) \leq \frac{1}{R^{2}} \mathbb{E}\left\|Y_{n}\right\|_{\gamma, 2, W, V}^{2} \leq \frac{C}{R^{2}} .
$$

Then given $\delta>0$, we can choose $R_{\delta}$ big enough such that

$$
P\left(Y_{n} \notin K_{R_{\delta}}\right) \leq \frac{C}{R_{\delta}^{2}}<\frac{\delta}{2}
$$

On the other hand, for any $L>0$, the set

$$
\widetilde{K}_{L}:=\left\{y \in C\left([0, T], \mathbb{R}^{m}\right): \sup _{t, s \in[0, T],|t-s|<\frac{T}{2^{M}}}|y(t)-y(s)| \leq \frac{L}{2^{\frac{M}{8}}}, \quad \forall M \in \mathbb{N}\right\}
$$


is compact in $C\left([0, T], \mathbb{R}^{m}\right)$. From the properties of the Brownian motion, we have

$$
P\left(\mathcal{W} \notin \widetilde{K}_{L}\right) \leq \frac{C}{L^{4}} .
$$

Hence given $\delta>0$, we can choose $L_{\delta}$ big enough such that

$$
P\left(\mathcal{W} \notin \widetilde{K}_{L_{\delta}}\right) \leq \frac{C}{L_{\delta}^{4}}<\frac{\delta}{2} .
$$

Therefore, there exists a relatively compact set $K_{R_{\delta}} \times \widetilde{K}_{L_{\delta}} \in \mathcal{B}\left(\mathcal{E} \times C\left([0, T], \mathbb{R}^{m}\right)\right)$ such that $\mu_{n}\left(K_{R_{\delta}} \times \widetilde{K}_{L_{\delta}}\right) \geq 1-\delta, \forall n \in \mathbb{N}$, which gives the tightness of the family $\left\{\mu_{n}\right\}$ on the space $\mathcal{E} \times$ $C\left([0, T], \mathbb{R}^{m}\right)$. Similar analysis can be found in $[27,28]$. It follows from the Prokhorov's Theorem that there exists a subsequence $\left\{\mu_{n_{k}}\right\}$ of $\left\{\mu_{n}\right\}$ which converges weakly to a probability measure $\mu$.

\subsubsection{Passage to the Limit}

Step 1. The Skorohod's Theorem provides the existence of a probability space $(\bar{\Omega}, \overline{\mathcal{F}}, \bar{P})$, and random variables $\left(\bar{Y}_{n_{k}}, \overline{\mathcal{W}}_{n_{k}}\right),(\bar{Y}, \overline{\mathcal{W}})$ defined on this probability space, with values in the space

$$
\mathcal{E} \times C\left([0, T], \mathbb{R}^{m}\right)
$$

such that:

(i) the law of $\left(\bar{Y}_{n_{k}}, \overline{\mathcal{W}}_{n_{k}}\right)$ is $\mu_{n_{k}}$,

(ii) the law of $(\bar{Y}, \overline{\mathcal{W}})$ is $\mu$,

(iii)

$$
\text { a.e. }-\bar{P}, \quad \text { we have } \quad\left(\bar{Y}_{n_{k}}, \overline{\mathcal{W}}_{n_{k}}\right) \rightarrow(\bar{Y}, \overline{\mathcal{W}}) \text { on } \mathcal{E} \times C\left([0, T], \mathbb{R}^{m}\right)
$$

To simplify the notation, we denote $Y_{k}:=Y_{n_{k}}, \bar{Y}_{k}:=\bar{Y}_{n_{k}}$ and $\overline{\mathcal{W}}_{k}:=\overline{\mathcal{W}}_{n_{k}}$.

Let us define the filtration $\left\{\overline{\mathcal{F}}_{t}\right\}_{t \in[0, T]}$ with

$$
\overline{\mathcal{F}}_{t}:=\sigma(Y(\lambda), \overline{\mathcal{W}}(\lambda), \quad 0 \leq \lambda \leq t) .
$$

Standard arguments show that the stochastic process $\overline{\mathcal{W}}(t), t \in[0, T]$, defined on the stochastic basis $\left(\bar{\Omega}, \overline{\mathcal{F}}, \bar{P},\left\{\overline{\mathcal{F}}_{t}\right\}_{t \in[0, T]}\right)$ is a Wiener process.

Let us consider

$$
f\left(Y_{k}\right):=v \Delta Y_{k}-\left(Y_{k} \cdot \nabla\right) v_{k}-\sum_{j} v_{k}^{j} \nabla Y_{k}^{j}+\left(\alpha_{1}+\alpha_{2}\right) \operatorname{div}\left(A_{k}^{2}\right)+\beta \operatorname{div}\left(\left|A_{k}\right|^{2} A_{k}\right),
$$

with $v_{k}=v\left(Y_{k}\right)$ and $A_{k}=A\left(Y_{k}\right)$.

Since $Y_{k}$ satisfies the equation

$$
\begin{aligned}
\left(v\left(Y_{k}(t)\right), e_{i}\right) & =\left(v\left(Y_{0}\right), e_{i}\right)+\int_{0}^{t}\left(f\left(Y_{k}\right), e_{i}\right) d s+\int_{0}^{t}\left(F\left(s, Y_{k}\right), e_{i}\right) d s \\
& +\int_{0}^{t}\left(\sigma\left(s, Y_{k}\right), e_{i}\right) d \mathcal{W}(s), \quad \forall i \in \mathbb{N},
\end{aligned}
$$

where the term $\int_{0}^{t}\left(\left(Y_{k} \cdot \nabla\right) v_{k}, e_{i}\right) d s$ is undestood as $\int_{0}^{t}\left(\left(Y_{k} \cdot \nabla\right) e_{i}, v_{k}\right) d s$. 
Step 2. It can be verified that $\bar{Y}_{k}$ satisfies the following stochastic differential equation

$$
\begin{aligned}
\left(v\left(\bar{Y}_{k}(t)\right), e_{i}\right) & =\left(v\left(Y_{0}\right), e_{i}\right)+\int_{0}^{t}\left(f\left(\bar{Y}_{k}\right), e_{i}\right) d s+\int_{0}^{t}\left(F\left(s, \bar{Y}_{k}\right), e_{i}\right) d s \\
& +\int_{0}^{t}\left(\sigma\left(s, \bar{Y}_{k}\right), e_{i}\right) d \overline{\mathcal{W}}_{k}(s), \quad \forall i \in \mathbb{N}
\end{aligned}
$$

with

$$
\int_{0}^{t}\left(\left(\bar{Y}_{k} \cdot \nabla\right) \bar{v}_{k}, e_{i}\right) d s:=\int_{0}^{t}\left(\left(\bar{Y}_{k} \cdot \nabla\right) e_{i}, \bar{v}_{k}\right) d s .
$$

Here we denote $\bar{v}_{k}=v\left(\bar{Y}_{k}\right), \bar{A}_{k}=A\left(\bar{Y}_{k}\right)$

Step 3. Taking into account that $\bar{Y}_{k}$ verifies Equation (30), following the same reasoning as in the proof of Lemma 3, we show that

$$
\mathbb{E}^{\bar{P}} \sup _{t \in[0, T]}\left\|\bar{Y}_{k}\right\|_{W}^{p} \leq C \quad \text { for } \quad 2 \leq p<\infty
$$

with $C$ independent of $k$, which gives

$$
\mathbb{E}^{\bar{P}}\left\|\bar{Y}_{k}\right\|_{L^{2}\left(0, T ; W^{1, q}\right)}^{p} \leq C \text { for } 2 \leq p, q<\infty,
$$

with $C$ independent of $k$. Since $\bar{Y}_{k} \rightarrow \bar{Y}$ a.e. $-\bar{P}$ strongly in $L^{2}\left(0, T ; W^{1, q}(\mathcal{O})\right)$ for $2 \leq q \leq 6$, we have

$$
\left\|\bar{Y}_{k}\right\|_{L^{2}\left(0, T ; W^{1, q}\right)}^{p} \rightarrow\|\bar{Y}\|_{L^{2}\left(0, T ; W^{1, q}\right)}^{p} \text { a.e. }-\bar{P}, \quad 2 \leq q \leq 6, \quad 2 \leq p<\infty,
$$

then the Fatou's Lemma yields

$$
\mathbb{E}^{\bar{P}}\|\bar{Y}\|_{L^{2}\left(0, T ; W^{1, q}\right)}^{p} \leq C \quad \text { for } \quad 2 \leq q \leq 6, \quad 2 \leq p<\infty .
$$

Therefore, the sequence $\left\{\left\|\bar{Y}_{k}-\bar{Y}\right\|_{L^{2}\left(0, T ; W^{1, q}\right)}^{p}\right\}$ is uniformly integrable and converges to zero in probabilty. As a consequence, we obtain

$$
\bar{Y}_{k} \rightarrow \bar{Y} \text { strongly in } L^{p}\left(\bar{\Omega}, L^{2}\left(0, T ; W^{1, q}(\mathcal{O})\right)\right) \text { for } \quad 2 \leq q \leq 6, \quad 2 \leq p<\infty .
$$

There exists a subsequence of $\left\{Y_{k}\right\}$, still denoted by $\left\{Y_{k}\right\}$ such that

$$
\bar{Y}_{k} \rightarrow \bar{Y} \text { strongly in } W^{1, q}(\mathcal{O}) \text {, a.e. }-d t \times \bar{P}, \quad \text { for } \quad 2 \leq q \leq 6 .
$$

In addition, from (31) we also have

$$
\bar{Y}_{k} \rightarrow \bar{Y} \quad \text { weakly- } * \text { in } \quad L^{p}\left(\bar{\Omega}, L^{\infty}(0, T ; W)\right) \text { for } 2 \leq p<\infty .
$$

Step 4. With the help of these two convergences, we will pass to the limit termwise in the stochastic differential Equation (30) in order to show that for all $t \in[0, T]$ and a.e. $-\bar{P}$, the stochastic process $\bar{Y}$ verifies the equation

$$
\begin{aligned}
(v(\bar{Y}(t)), \phi) & =\left(v\left(Y_{0}\right), \phi\right)+\int_{0}^{t}(f(\bar{Y}), \phi) d s+\int_{0}^{t}(F(s, \bar{Y}), \phi) d s \\
& +\int_{0}^{t}(\sigma(s, \bar{Y}), \phi) d \overline{\mathcal{W}}(s), \quad \forall \phi \in V .
\end{aligned}
$$

Let $e_{i}, i \in \mathbb{N}$, an arbitrary element of the basis. Due to the convergence (35), we have

$$
\left(v\left(\bar{Y}_{k}(t)\right), e_{i}\right)=\left(\bar{Y}_{k}(t), e_{i}\right)_{V} \rightarrow\left(\bar{Y}(t), e_{i}\right)_{V}=\left(v(Y(t)), e_{i}\right), \quad \text { a.e. }-d t \times \bar{P} .
$$


Let us write

$$
\begin{aligned}
\int_{0}^{t}\left(f\left(\bar{Y}_{k}\right), e_{i}\right) d s & =v \int_{0}^{t}\left(\Delta \bar{Y}_{k}, e_{i}\right) d s-\int_{0}^{t}\left(\left(\bar{Y}_{k} \cdot \nabla\right) \bar{v}_{k}, e_{i}\right) d s-\int_{0}^{t} \sum_{j}\left(\bar{v}_{k}^{j} \nabla \bar{Y} k^{j}, e_{i}\right) d s \\
& +\left(\alpha_{1}+\alpha_{2}\right) \int_{0}^{t}\left(\operatorname{div}\left(\bar{A}_{k}^{2}\right), e_{i}\right) d s+\beta \int_{0}^{t}\left(\operatorname{div}\left(\left|\bar{A}_{k}\right|^{2} \bar{A}_{k}\right), e_{i}\right) d s .
\end{aligned}
$$

For all test function $\xi \in L^{\infty}([0, T] \times \Omega)$, the weak convergence (36) gives

$$
\mathbb{E} \int_{0}^{T}\left(\Delta \bar{Y}_{k}, \xi e_{i}\right) d s \rightarrow \mathbb{E} \int_{0}^{T}\left(\Delta \bar{Y}, \xi e_{i}\right) d s .
$$

With the help of the convergences (34) and (36), we deduce that

$$
\begin{aligned}
\mathbb{E} \int_{0}^{T}\left(\left(\bar{Y}_{k} \cdot \nabla\right) \bar{v}_{k}, \xi e_{i}\right) d s=\mathbb{E} \int_{0}^{T}\left(\left(\bar{Y}_{k} \cdot \nabla\right) \xi e_{i}, \bar{v}_{k}\right) d s & \rightarrow \mathbb{E} \int_{0}^{T}\left((\bar{Y} \cdot \nabla) \xi e_{i}, \bar{v}\right) d s \\
& =\mathbb{E} \int_{0}^{T}\left((\bar{Y} \cdot \nabla) \bar{v}, \xi e_{i}\right) d s .
\end{aligned}
$$

Notice that the term $\operatorname{div}\left(\left|\bar{A}_{k}\right|^{2} \bar{A}_{k}\right)$ can be written in the form $\mathcal{D}^{2} \bar{Y}_{k} \mathcal{D} \bar{Y}_{k} \mathcal{D} \bar{Y}_{k}$ where $\mathcal{D}^{2}$ is a differential operator of second order, and $\mathcal{D}$ is a first order differential operator. The strong convergence (34) yields

$$
\mathcal{D} \bar{Y}_{k} \rightarrow \mathcal{D} \bar{Y} \quad \text { in } \quad L^{4}\left(\Omega, L^{2}\left(0, T ; L^{4}(\mathcal{O})\right)\right)
$$

that implies

$$
\mathcal{D} \bar{Y}_{k} \mathcal{D} \bar{Y}_{k} \rightarrow \mathcal{D} \bar{Y} \mathcal{D} \bar{Y} \quad \text { in } \quad L^{2}\left(\Omega, L^{1}\left(0, T ; L^{2}(\mathcal{O})\right)\right)
$$

On the other hand

$$
\mathcal{D}^{2} \bar{Y}_{k} \rightarrow \mathcal{D}^{2} \bar{Y} \quad \text { weakly-* in } \quad L^{2}\left(\Omega, L^{\infty}\left(0, T ; L^{2}(\mathcal{O})\right)\right) .
$$

Therefore, we have

$$
\begin{aligned}
\mathbb{E} \int_{0}^{T}\left(\operatorname{div}\left(\left|\bar{A}_{k}\right|^{2} \bar{A}_{k}\right), \xi e_{i}\right) d s=\mathbb{E} \int_{0}^{T}\left(\mathcal{D}^{2} \bar{Y}_{k} \mathcal{D} \bar{Y}_{k} \mathcal{D} \bar{Y}_{k}, \xi e_{i}\right) d s & \rightarrow \mathbb{E} \int_{0}^{T}\left(\mathcal{D}^{2} \bar{Y} \mathcal{D} \bar{Y} \mathcal{D} \bar{Y}, \xi e_{i}\right) d s \\
& =\mathbb{E} \int_{0}^{T}\left(\operatorname{div}\left(|\bar{A}|^{2} \bar{A}\right), \xi e_{i}\right) d s
\end{aligned}
$$

Dealing in a similar way with the remaing term, we reach the convergence

$$
\mathbb{E} \int_{0}^{T}\left(f\left(\bar{Y}_{k}\right), \xi e_{i}\right) d s \rightarrow \mathbb{E} \int_{0}^{T}\left(f(\bar{Y}), \xi e_{i}\right) d s,
$$

which yields

$$
\int_{0}^{t}\left(f\left(\bar{Y}_{k}\right), e_{i}\right) \rightarrow \int_{0}^{t}\left(f(\bar{Y}), e_{i}\right)
$$

weakly in $L^{1}(\Omega)$.

In addition, considering the assumption (17), we derive

$$
\mathbb{E}\left|\int_{0}^{t}\left(F\left(s, \bar{Y}_{k}\right)-F(s, \bar{Y}), e_{i}\right) d s\right| \leq C\left\|\bar{Y}_{k}-\bar{Y}\right\|_{L^{2}\left(\Omega, L^{2}(0, T ; V)\right)} \rightarrow 0 .
$$


For the stochastic term, we will verify that for each $t$

$$
\int_{0}^{t}\left(\sigma\left(s, \bar{Y}_{k}\right), e_{i}\right) d \overline{\mathcal{W}}_{k}(s) \rightarrow \int_{0}^{t}\left(\sigma(s, \bar{Y}), e_{i}\right) d \overline{\mathcal{W}}(s) \text { weakly in } L^{1}(\Omega) .
$$

We argue as in [27] (see also [28]), taking a regularization of the diffusion coefficient. For the sake of completeness, we sketch the main ideas. For each path $y:[0, T] \rightarrow V$, we define

$$
\sigma^{\delta}(\tau, y):[0, T] \rightarrow H, \quad \sigma^{\delta}(\tau, y):=\frac{1}{\delta} \int_{0}^{\tau} \mathrm{e}^{-\frac{(\tau-s)}{\delta}} \sigma(s, y(s)) d s .
$$

Since $\tau \rightarrow \sigma^{\delta}(\tau, y)$ is regular, we integrate by parts and write the stochastic integral as a function of the pair $\left(\bar{Y}_{k}, \overline{\mathcal{W}}_{k}\right)$ as follows

$$
\begin{aligned}
\int_{0}^{t}\left(\sigma^{\delta}\left(s, \bar{Y}_{k}\right), e_{i}\right) \overline{\mathcal{W}}_{k}(s) & =G\left(\bar{Y}_{k}, \overline{\mathcal{W}}_{k}\right) \\
& \left.=\sigma^{\delta}\left(t, \bar{Y}_{k}\right), e_{i}\right) \overline{\mathcal{W}}_{k}(t)-\int_{0}^{t} \frac{d}{d s}\left(\sigma^{\delta}\left(s, \bar{Y}_{k}\right), e_{i}\right) \overline{\mathcal{W}}_{k}(s) d s .
\end{aligned}
$$

In this expression, we use the convergence (28) in order to deduce that

$$
\int_{0}^{t}\left(\sigma^{\delta}\left(s, \bar{Y}_{k}\right), e_{i}\right) d \overline{\mathcal{W}}_{k}(s) \rightarrow \int_{0}^{t}\left(\sigma^{\delta}(s, \bar{Y}), e_{i}\right) d \overline{\mathcal{W}}(s) \text { a.e. }-\bar{P} .
$$

For any $\varphi \in L^{\infty}(\Omega)$ and each $\delta>0$, we apply the assumption (18) and Lemma 3 to obtain

$$
\mathbb{E}\left(\int_{0}^{t}\left(\sigma^{\delta}\left(s, \bar{Y}_{k}\right), \varphi e_{i}\right) d \overline{\mathcal{W}}_{k}(s)\right)^{2} \leq C \mathbb{E} \int_{0}^{t}\left\|\sigma^{\delta}\left(s, \bar{Y}_{k}\right)\right\|_{2}^{2} d s \leq C \mathbb{E} \int_{0}^{t}\left\|\sigma\left(s, \bar{Y}_{k}\right)\right\|_{2}^{2} d s \leq C, \quad \forall k \in \mathbb{N} ;
$$

then the sequence

$$
\left\{\int_{0}^{t}\left(\sigma^{\delta}\left(s, \bar{Y}_{k}\right), \varphi e_{i}\right) d \overline{\mathcal{W}}_{k}(s)\right\}
$$

is uniformly integrable, and consequently

$$
\int_{0}^{t}\left(\sigma^{\delta}\left(s, \bar{Y}_{k}\right), e_{i}\right) d \overline{\mathcal{W}}_{k}(s) \rightarrow \int_{0}^{t}\left(\sigma^{\delta}(s, \bar{Y}), e_{i}\right) d \overline{\mathcal{W}}(s) \quad \text { weakly in } \quad L^{1}(\Omega) .
$$

On the other hand, for each $k$ it holds that

$$
\int_{0}^{t}\left(\sigma^{\delta}\left(s, \bar{Y}_{k}\right), e_{i}\right) d \overline{\mathcal{W}}_{k}(s) \rightarrow \int_{0}^{t}\left(\sigma\left(s, \bar{Y}_{k}\right), e_{i}\right) d \overline{\mathcal{W}}_{k}(s),
$$

and

$$
\int_{0}^{t}\left(\sigma^{\delta}(s, \bar{Y}), e_{i}\right) d \overline{\mathcal{W}}(s) \rightarrow \int_{0}^{t}\left(\sigma(s, \bar{Y}), e_{i}\right) d \overline{\mathcal{W}}(s) \quad \text { in } \quad L^{2}(\Omega), \quad \text { as } \quad \delta \rightarrow 0 .
$$

Taking into account that

$$
\begin{aligned}
& \left|\mathbb{E} \int_{0}^{t}\left(\sigma\left(s, \bar{Y}_{k}\right), \varphi e_{i}\right) d \overline{\mathcal{W}}_{k}(s)-\mathbb{E} \int_{0}^{t}\left(\sigma(s, \bar{Y}), \varphi e_{i}\right) d \overline{\mathcal{W}}(s)\right| \\
& \leq\left|\mathbb{E} \int_{0}^{t}\left(\sigma\left(s, \bar{Y}_{k}\right), \varphi e_{i}\right) d \overline{\mathcal{W}}_{k}(s)-\mathbb{E} \int_{0}^{t}\left(\sigma^{\delta}\left(s, \bar{Y}_{k}\right), \varphi e_{i}\right) d \overline{\mathcal{W}}_{k}(s)\right| \\
& +\left|\mathbb{E} \int_{0}^{t}\left(\sigma^{\delta}\left(s, \bar{Y}_{k}\right), \varphi e_{i}\right) d \overline{\mathcal{W}}_{k}(s)-\mathbb{E} \int_{0}^{t}\left(\sigma^{\delta}(s, \bar{Y}), \varphi e_{i}\right) d \overline{\mathcal{W}}(s)\right| \\
& +\left|\mathbb{E} \int_{0}^{t}\left(\sigma^{\delta}(s, \bar{Y}), \varphi e_{i}\right) d \overline{\mathcal{W}}(s)-\mathbb{E} \int_{0}^{t}\left(\sigma(s, \bar{Y}), \varphi e_{i}\right) d \overline{\mathcal{W}}(s)\right|,
\end{aligned}
$$


and using (39)-(41), we deduce that

$$
\int_{0}^{t}\left(\sigma\left(s, \bar{Y}_{k}\right), e_{i}\right) d \overline{\mathcal{W}}_{k}(s) \rightarrow \mathbb{E} \int_{0}^{t}\left(\sigma(s, \bar{Y}), e_{i}\right) d \overline{\mathcal{W}}(s) \quad \text { weakly in } \quad L^{1}(\Omega) .
$$

With the help of all deduced convergence results, we are able to pass to the limit each term of Equation (30), showing that the stochastic process $\bar{Y}$ satisfies Equation (37) for $\phi=e_{i}, \forall i \in \mathbb{N}$. Since $\operatorname{span}\left\{e_{1}, \ldots, e_{n}, \ldots\right\}=V$, we conclude that $\bar{Y}$ satisfies Equation (37) for all $\phi \in V$. Hence the system $\left(\bar{\Omega}, \overline{\mathcal{F}}, \bar{P},\left\{\overline{\mathcal{F}}_{t}\right\}_{t \in[0, T]}, \overline{\mathcal{W}}, \bar{Y}\right)$ is a solution of the equation.

\section{Discussion and Methods}

In this article, we studied the third grade fluid equations in the three-dimensional physical space, perturbed by a multiplicative white noise. We established the existence of a weak stochastic solution, where the velocity field corresponds to a stochastic process defined on a certain probability space with sample paths in $L^{\infty}\left(0, T ; H^{2}(\mathcal{O})\right)$, and the Wiener process is part of the solution.

We should mention that there are a few results in the literature on the stochastic third grade fluid equations. The two-dimensional stochastic third grade fluid equations was recently studied in [18], where the authors proved the existence and the uniqueness of the strong solution (in the stochastic sense) by using a unicity type argument. In [23] it is proved that the strong two-dimensional solution constructed in [18] satisfies a large deviation principle. As far as we know, the stochastic three-dimensional problem is being studied here for the first time. Since the unicity type arguments and the compactness strategy applied in dimension two do not work in dimension three; here, we followed a more probabilistic approach based on the application of a tightness criterion combined with the Prokhorov's and Skorohod's Theorems. The uniqueness in three dimensions is not an easy issue and remains an open problem.

We expect that our approach and our result will have practical consequences, contributing to the statistical description of non-Newtonian turbulent fluids, where typically the flows possess lack of regularity in the time and space variables. In a forthcoming paper, we intend to show the existence of less regular solutions by taking the initial conditions in $H^{1}(\mathcal{O})$, and to study the corresponding moments and correlation functions. A relevant direction for future research concerns the existence of stationary statistical solutions, or invariant measures.

\section{Conclusions}

In this work, we considered the equations for an incompressible third grade fluid on a non-axisymmetric and simply connected bounded domain $\mathcal{O} \subset \mathbb{R}^{3}$ with a sufficiently regular boundary $\Gamma$, supplemented with a Navier slip boundary condition, and perturbed by a multiplicative white noise of Itô type. Taking initial conditions in the Sobolev space $H^{2}(\mathcal{O})$, we proved the existence of a solution in the space $L^{\infty}\left(0, T ; H^{2}(\mathcal{O})\right)$, in the weak probabilistic sense.

Author Contributions: Conceptualization A.A. and F.C.; investigation A.A. and F.C.; formal analysis A.A. and F.C.; writing-original draft preparation A.A. and F.C.; writing-review and editing A.A. and F.C. Both authors have read and agreed to the published version of the manuscript.

Funding: This research received no external funding.

Acknowledgments: We would like to thank the anonymous referees for relevant suggestions and comments which contributed to improve the article. The authors are very grateful to the institutions Fundação Calouste Gulbenkian and Fundação para a Ciência e a Tecnologia due to financial support. The work of A. Almeida was supported by the Calouste Gulbenkian Foundation, through the Scholarship Program for students from the PALOP and Timor-Leste. The work of F. Cipriano was supported by the Fundação para a Ciência e a Tecnologia (Portuguese Foundation for Science and Technology) through the project UID/MAT/00297/2019 (Centro de Matemática e Aplicações).

Conflicts of Interest: The authors declare no conflict of interest. 


\section{References}

1. Fosdick, R.L.; Rajagopal, K.R. Thermodynamics and stability of fuids of third grade. Proc. R. Soc. Lond. Ser. A 1980, 339, 351-377. [CrossRef]

2. Dunn, J.E.; Rajagopal, K.R. Fluids of differential type: Critical review and thermodynamical analysis. Int. J. Eng. Sci. 1995, 33, 689-729. [CrossRef]

3. Hayat, T.; Kara, A.H.; Momoniat, E. Exact flow of a third-grade fluid on a porous wall. Int. J. Non Linear Mech. 2003, 38, 1533-1537. [CrossRef]

4. Hayat, T.; Shahzad, F.; Ayub, M. Analytical solution for the steady flow of the third grade fluid in a porous half space. Appl. Math. Model. 2007, 31, 2424-2432. [CrossRef]

5. Rasheed, A.; Kausar, A.; Wahab, A.; Akbar, T. Stabilized approximation of steady flow of third grade fluid in presence of partial slip. Res. Phys. 2017, 7, 3181-3189. [CrossRef]

6. Rivlin, R.S.; Ericksen, J.L. Stress-deformation relations for isotropic materials. Arch. Ration. Mech. Anal. 1955, 4, 323-425. [CrossRef]

7. Wang, S.-Q.; Drda, P.A. Stick-slip transition in capillary flow of linear polyethylene: 3. Surface conditions. Rheol. Acta 1997. [CrossRef]

8. Meeker, S.P.; Bonnecaze, R.T.; Cloitre, M. Slip and flow in pastes of soft particles: Direct observation and rheology. J. Rheol. 2004, 84, 1295. [CrossRef]

9. Foias, C.; Manley, O.; Rosa, R.; Temam, R. Navier-Stokes Equations and Turbulence; Cambridge University Press: Cambridge, UK, 2001.

10. McComb, W.D. The Physics of Fluid Turbulence; Oxford University Press: New York, NY, USA, 1992.

11. Monin, A.S.; Yaglom, A.M. Statistical Fluid Mechanics: Mechanics of Turbulence, Vol. I; The MIT Press: Cambridge, MA, USA, 1979.

12. Monin, A.S.; Yaglom, A.M. Statistical Fluid Mechanics: Mechanics of Turbulence, Vol. II; The MIT Press: Cambridge, MA, USA, 1981.

13. Holm, D.D. Variational principles for stochastic fluid dynamics. Proc. R. Soc. A 2015, 471, 20140963. [CrossRef] [PubMed]

14. Holm, D.D.; Tyranowski, T.M. Variational principles for stochastic soliton dynamics. Proc. R. Soc. A 2016, 472, 20150827. [CrossRef] [PubMed]

15. Chemetov, N.V.; Cipriano, F. Well-posedness of stochastic second grade fluids. J. Math. Anal. Appl. 2017, 457, 585-616. [CrossRef]

16. Chemetov, N.V.; Cipriano, F. Optimal control for two-dimensional stochastic second grade fluids. Stoch. Proc. Their Appl. 2018, 128, 2710-2749. [CrossRef]

17. Razafimandimby, P.A.; Sango, M. Strong solution for a stochastic model of two-dimensional second grade fluids: Existence, uniqueness and asymptotic behavior. Nonlinear Anal. 2012, 75, 4251-4270. [CrossRef]

18. Cipriano, F.; Didier, F.; Guerra, S. Well-posedness of stochastic third grade fluid equation. J. Differ. Equ. 2020, under review.

19. Busuioc, A.V.; Iftimie, D. Global existence and uniqueness of solutions for the equations of third grade fluids. Int. J. Non Linear Mach. 2004, 39, 1-12. [CrossRef]

20. Busuioc, A.V.; Iftimie, D. A non-Newtonian fluid with Navier boundary conditions. J. Dynam. Diff. Equ. 2006, 18, 357-379. [CrossRef]

21. Breckner, H.I. Approximation and Optimal Control of the Stochastic Navier-Stokes Equation. Ph.D. Thesis, Martin-Luther University, Halle, Germany, 1999.

22. Simon, J. Compact Sets in the Space $L^{p}(0, T ; B)$. Ann. Mat. Pura Appl. 1986, 146, 65-96. [CrossRef]

23. Almeida, A.; Cipriano, F. A large deviation principle for fluids of third grade. J. Math. Anal. Appl. 2020, under review.

24. Busuioc, A.V.; Ratiu, T.S. The second grade fluid and averaged Euler equations with Navier-slip boundary conditions. Nonlinearity 2003, 16, 1119-1149. [CrossRef]

25. Albeverio, S.; Brzeźniak, Z.; Wu, J.-L. Existence of global solutions and invariant measures for stochastic differential equations driven by Poisson type noise with non-Lipschitz coefficients. J. Math. Anal. Appl. 2010, 371, 142-149. [CrossRef]

26. Desvillettes, L.; Villani, C. On a variant of Korn's inequality arising in statistical mechanics. ESAIM Control Optim. Calc. Var. 2002, 8, 603-619. [CrossRef] 
27. Bensoussan, A. Stochastic Navier-Stokes equations. Acta Appl. Math. 1995, 38, 267-304. [CrossRef]

28. Razafimandimby, P.A.; Sango, M. Weak solutions of a stochastic model for two-dimensional second grade fluids. Bound. Value Probl. 2010, 2010, 636140. [CrossRef]

Publisher's Note: MDPI stays neutral with regard to jurisdictional claims in published maps and institutional affiliations.

(C) 2020 by the authors. Licensee MDPI, Basel, Switzerland. This article is an open access article distributed under the terms and conditions of the Creative Commons Attribution (CC BY) license (http://creativecommons.org/licenses/by/4.0/). 